\title{
Electrical stimulation in the lateral hypothalamus in rats in the activity-based anorexia model
}

\author{
Laboratory investigation
}

\author{
Marleen Welkenhuysen M.Sc., ${ }^{1}$ Kris van Kuyck, Ph.D., ${ }^{1}$ John Das, B.S., ${ }^{1}$ \\ Raf Sciot, M.D., Ph.D., ${ }^{2}$ And Bart NutTin, M.D., Ph.D. ${ }^{1}$ \\ ${ }^{1}$ Laboratory of Experimental Functional Neurosurgery, Department of Neuroscience; and \\ ${ }^{2}$ Department of Morphology and Molecular Pathology Section, Katholieke Universiteit Leuven, \\ Provisorium I, Leuven, Belgium
}

\begin{abstract}
Object. One quarter of patients with anorexia nervosa have a poor outcome and continue to suffer chronically or die. Electrical brain stimulation may be of therapeutic benefit in some of these patients; however, the brain target for inducing symptom relief is unknown. In this study, the authors evaluated the effects of acute and chronic electrical stimulation in the lateral hypothalamus on food intake, locomotor activity, and survival time in rats in an activity-based anorexia model.

Methods. In an acute experiment, the authors electrically stimulated at $100 \mathrm{~Hz}$ and $0,25,50$ and $75 \%$ of the maximal stimulation amplitude (that is, the amplitude leading to severe side effects) in the lateral hypothalamus on consecutive days during 4 test sessions in 10 rats and evaluated food intake and locomotor activity. In a chronic experiment, they compared food intake, wheel revolutions, and survival time between 6 rats that underwent electrical stimulation in the lateral hypothalamus (50\% of maximal stimulation amplitude) and 8 rats that did not undergo stimulation.

Results. In the acute experiment, overall electrical stimulation $(25,50$, and $75 \%$ combined) and stimulation at $75 \%$ of the maximal stimulation amplitude significantly decreased the locomotor activity. However, if the authors omitted results of 1 rat, in which the electrode tip was not located in the lateral hypothalamus on one side but rather in the supraoptic chiasm, the remaining results did not yield significance. No other differences were observed.

Conclusions. When the findings of the current study are extrapolated to patients with anorexia nervosa, the authors do not expect major effects on symptoms with electrical stimulation at high frequency in the lateral hypothalamus. (DOI: 10.3171/FOC/2008/25/7/E7)
\end{abstract}

\section{KEY WORDS • activity-based anorexia • anorexia nervosa • electrical brain stimulation - lateral hypothalamus}

$\mathrm{A}$ NOREXIA nervosa is a severe psychiatric disorder with a prevalence up to $0.9 \%$ among young females, and according to the criteria of the Diagnostic and Statistical Manual of Mental Disorders-IV the average of the European studies is $0.29 \% .^{18}$ The incidence in a large representative sample in the Netherlands $(1.1 \%$ of total population) was 8.1 per 100,000 per year. Females are affected more than males, and the female-to-male ratio is $>$ 10:1. The 15- to 19-year age group represents the highest rate, constituting $\sim 40 \%$ of all identified cases.

Despite intensive treatment programs (for example, with psycho- and/or pharmacotherapy), prospective long-term follow-up investigations reveal a poor outcome in a quarter of the patients. These patients continue to suffer or die due to suicide or physical complications associated with extreme starvation like dehydration and electrolyte imbal- ance. The mortality rates have been reported to be 3 and $15.6 \%$ after 5 and 21 years of follow-up, respectively., ${ }^{5,50}$

Functional neuroimaging and neuroendocrinological studies have provided substantial evidence for the involvement of the central nervous system in the mediation and maintenance of the symptoms in patients with anorexia nervosa. ${ }^{45}$ We therefore speculate that therapeutic interventions directed at the central nervous system, like electrical brain stimulation, may alleviate symptoms in patients suffering from treatment-resistant anorexia nervosa. Likewise, electrical brain stimulation has proven successful in patients suffering from treatment-resistant obsessive-compulsive disorder and major depression disorder, 2 psychiatric disorders related to anorexia nervosa. ${ }^{25,29-32}$ Because it is unknown in which brain targets electrical stimulation relieves symptoms, we rely on the activity-based anorexia 
rat model to predict outcome of electrical stimulation in patients with anorexia nervosa.

In this model, the food intake of rats that can eat at libitum during 1.5 hours daily for several weeks is enough to maintain body weight. However, in the presence of a running wheel in the cage, these rats will display hyperactivity after 7 to 10 days and spontaneously restrict their food intake leading to severe emaciation and often even death. ${ }^{40}$ This behavior models some of the symptoms in patients with anorexia nervosa: dieting and excessive exercising. ${ }^{21}$ However, we are well aware that some other core symptoms, like the patient's disturbance in the perception of body weight and shape, and the intense fear of gaining weight or becoming fat, are likely not present in the activity-based anorexia model.

The lateral hypothalamus plays a key role in the regulation of food intake and body weight homeostasis. ${ }^{6}$ In an acute experiment in rats in the activity-based anorexia model, we stimulated at $0,25,50$ and $75 \%$ of the maximal stimulation amplitude, that is, the amplitude leading to severe side effects. We hypothesized that overall electrical stimulation $(25,50$, and $75 \%$ combined) and stimulation at $75 \%$ of the maximal stimulation amplitude would reduce activity and increase food intake. Moreover, we hypothesized that the changes are stimulation-amplitude dependent. In a chronic experiment in rats in the activity-based anorexia model, we stimulated at 0 and $50 \%$ of the maximal stimulation amplitude, and hypothesized that stimulation at $50 \%$ of the maximal stimulation amplitude decreases hyperactivity, and increases food intake and survival time in rats in the activity-based anorexia model.

\section{Methods}

\section{Test Animals}

Twenty-six female albino Wistar rats, weighing 200$250 \mathrm{~g}$ at the start of the experiment, were housed 2 per cage before surgery and individually after surgery. They had free access to food before the start of the experiments in the activity-based anorexia model and to water at all times. All experiments were carried out in accordance with protocols approved by the local university animal ethics committee and in accordance with the European Communities Council Directive of November 24, 1986 (86/609/EEC).

\section{Experimental Cages}

Activity-based anorexia experiments were conducted in custom-made cages $(0.36 \times 0.36 \times 0.36 \mathrm{~m})$ with a running wheel (diameter $0.35 \mathrm{~m}$; Campden Instruments). An electromagnetic rotary encoder (TWK-Elektronik $\mathrm{GmbH}$ ) was mounted on the shaft of the running wheel for continuous registration of the position of the running wheel with a digital I/O card (National Instruments) and LabVIEW 7.0 (National Instruments).

\section{Surgical Procedure}

Amoxicillin (subcutaneous $0.4 \mathrm{ml}$; Duphamox, Intervet International B.V.) was prophylactically injected. After induction of general anesthesia (subcutaneous $400 \mathrm{mg} / \mathrm{kg}$ chloral hydrate; Sigma-Aldrich) the head was positioned in a stereotactic frame (Narishige) with the bregma and lamb- da positioned in the horizontal plane. Lidocaine (subcutaneous $0.4 \mathrm{ml}$ Xylocaine $2 \% 20 \mathrm{mg} / \mathrm{ml}$, AstraZeneca SA) was administered locally before incising the skin. Monopolar $80 \%$ platinum $/ 20 \%$ iridium stimulation electrodes (Goodfellow Cambridge Ltd.) with a diameter of $200 \mu \mathrm{m}$ and a bare transversally cut tip were implanted bilaterally in the lateral hypothalamus $(2.6 \mathrm{~mm}$ posterior to the bregma, $1.8 \mathrm{~mm}$ lateral to the midline, and $8.3 \mathrm{~mm}$ ventral to the dural surface) using the coordinates of the atlas of Paxinos and Watson. ${ }^{34}$ Self-tapping bone screws (Fine Science Tools) were inserted in the skull and served as reference electrodes and as anchor points to mount the cement block. This cement block kept the electrodes in position and externalized the connectors, which conducted the current in case of electrical stimulation.

\section{Study Design}

Acute Experiment. One week after implantation of the electrodes, 10 rats were placed in the activity-based anorexia test cages. When body weight decreased to $85 \%$ of the initial body weight (that is, the body weight when the animal was first placed in the experimental cage), the maximal stimulation amplitude was determined, and during subsequent days 4 test sessions were performed in random order with stimulation amplitude (independent variable) at 0,25 , 50 , and $75 \%$ of the maximal stimulation amplitude. The dependent variables in the acute experiment were the number of revolutions in the running wheel during the 2 hours preceding the feeding period (9-11 a.m.) and food intake during the 1.5 -hour feeding period. Body weight was measured every day, and a limited amount of extra food was provided daily after the test sessions to keep body weight at $85 \%$ of the initial body weight $(1 \mathrm{~g}$ of food for every $1 \%$ below $85 \%$ of the initial body weight). The observer, who measured food intake and body weight, was blinded to the test condition.

Chronic Experiment. One week after implantation of the electrodes, rats were randomly allocated to the stimulationon or -off group (8 rats/group) and subsequently placed in the experimental cages. When body weight decreased to $85 \%$ of the initial body weight, the maximal stimulation amplitude was determined in the stimulation-on group, and stimulation was started at $50 \%$ of the maximal stimulation amplitude in this group. This stimulation amplitude was chosen because minor side effects were sometimes observed at $75 \%$ of the maximal stimulation amplitude. The stimulation-off group was handled in the same manner, but electrical stimulation was not applied. The dependent variables in the chronic experiment were the daily number of rotations (measured continuously) and daily food intake during the 1.5-hour feeding period. In addition, we measured the number of days until the animal died or until body weight decreased to $70 \%$ of the initial body weight. When this occurred, experiments were terminated for that individual rat. Experiments were also terminated in case body weight remained constant or increased during 5 consecutive days $(<1 \%$ decrease in body weight compared with the previous day); the rat was considered not susceptible for activity-based anorexia. Rats that did not die were killed with an overdose of Nembutal. The observer, who measured food intake and body weight, was blinded to the test condition. 

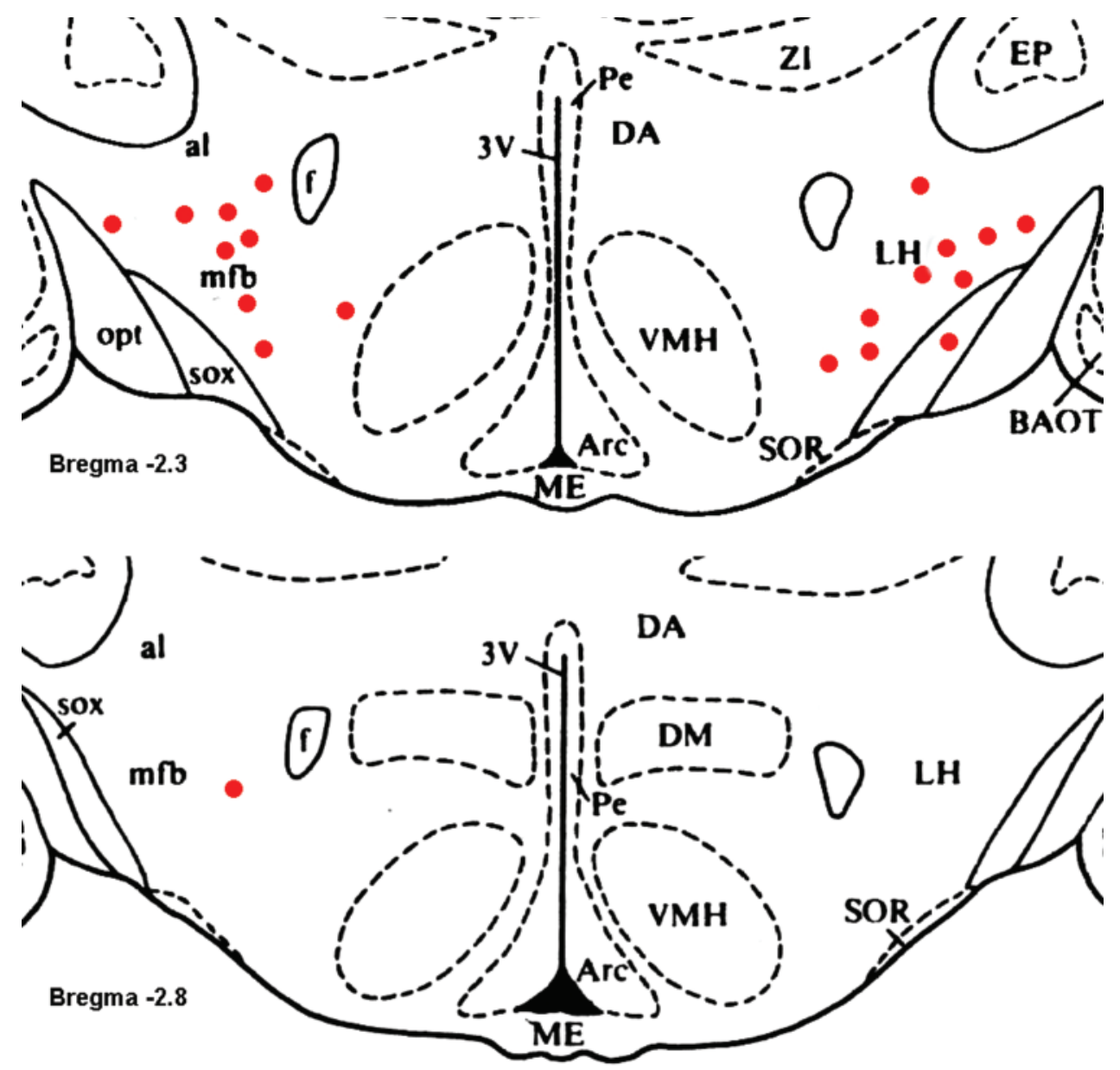

FIG. 1. Schematic representation of the position of the electrode tips in the acute experiment. Coronal sections 2.3 and $2.8 \mathrm{~mm}$ posterior to bregma (Paxinos and Watson). Note that in 1 of the animals, the electrode tip is unilaterally located in the supraoptic chiasm (sox). $\mathrm{Al}=$ ansa lenticularis; arc $=$ arcuate hypothalamic nucleus; $\mathrm{BAOT}=$ bed nucleus of the accessory olfactory tract; $\mathrm{DA}=$ dorsal hypothalamic area; $\mathrm{DM}=$ dorsomedial hypothalamic nucleus; $\mathrm{EP}=$ entopeduncular nucleus; $\mathrm{f}=$ fornix; $\mathrm{LH}=$ lateral hypothalamus; $\mathrm{ME}=$ medial amygdaloid nucleus; $\mathrm{mfb}=$ medial forebrain bundle; $\mathrm{opt}=$ optic tract; $\mathrm{Pe}=$ periventricular hypothalamic; $\mathrm{SOR}=$ supraoptic hypothalamic nucleus retrochiasmatic; $\mathrm{VMH}=$ ventromedial hypothalamic nucleus; ZI = zona incerta. Reprinted with permission from Paxinos G, Watson C: The Rat Brain in Stereotactic Coordinates, ed 2. Sydney: Academic Press, 1986

\section{Electrical Stimulation}

Acute Experiment. Constant current, biphasic, cathodicfirst square wave pulses with a pulse width of $0.06 \mathrm{msec}(1$ unidirectional phase) were administered at a frequency of $100 \mathrm{~Hz}$ starting 2 hours before the food intake period and lasting 3.5 hours in total ( 9 a.m. -12.30 p.m.). The maximal stimulation amplitude (in $\mathrm{mA}$ ) was the amplitude that clearly interfered with normal behavior.

Chronic Experiment. Electrical stimulation parameters were identical to those in the acute experiment except that electrical stimulation was applied continuously starting when the rat's body weight decreased to lower than $85 \%$ of the initial body weight until the end of the experiment.

\section{Histological Analysis}

After the experiments, the rats were killed with an overdose of pentobarbital (intraperitoneal $3 \mathrm{ml}$ Nembutal, CEVA Santé Animal) and the brains were removed and quickly frozen at $\left(-40^{\circ} \mathrm{C}\right.$ in 2-methylbutane (106056, Merck KgaA). Verification of the electrode tip location was performed in 25- $\mu \mathrm{m}$ cryostat sections stained with thionin (Janssen Chimica). The electrode tip was localized based on the atlas of Paxinos and Watson, ${ }^{34}$ taking into account the surrounding anatomy.

\section{Data Analysis}

Acute Experiment. Repeated measurement analysis was used to unravel the overall effect of electrical stimulation in the lateral hypothalamus on the number of rotations and food intake. Because data regarding the number of rotations and food intake at $75 \%$ of the maximal stimulation amplitude were missing in 1 animal, the procedure PROC MIXED was used instead of PROC GLM in the statistical software (version 9.1.2, SAS Institute Inc.). The paired Wilcoxon test was used to determine the effect of electrical stimulation at 75 versus $0 \%$ of the maximal stimulation amplitude. 

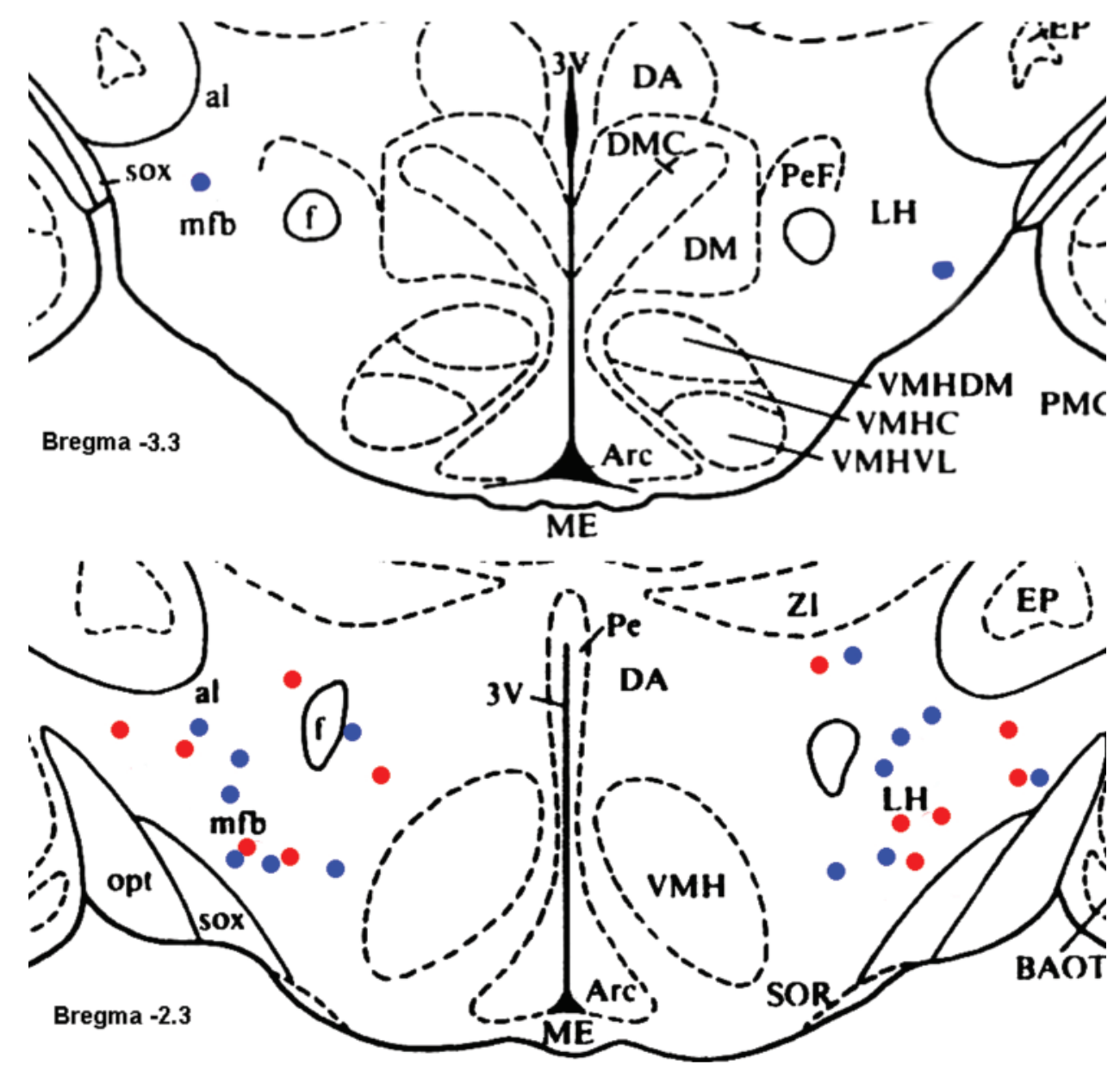

FIG. 2. Schematic representation of the position of the electrode tips of the experimental (red dots) and control group (blue dots) in the chronic experiment. Coronal sections 2.3 and $3.3 \mathrm{~mm}$ posterior to bregma. DMC $=$ dorsomedial hypothalamic nucleus, compact part; $\mathrm{PeF}=$ perifornical hypothalamic nucleus; $\mathrm{PMCo}=$ posteromedial cortical amygdaloid nucleus; VMHDM = ventromedial hypothalamic nucleus, dorsomedial part; VMHC = ventromedial hypothalamic nucleus, central part; VMHVL = ventromedial hypothalamic nucleus, ventrolateral part. Reprinted with permission from Paxinos G, Watson C: The Rat Brain in Stereotactic Coordinates, ed 2. Sydney: Academic Press, 1986.

Chronic Experiment. We searched for differences in the number of days until the animal died (or until body weight decreased to $70 \%$ of the initial body weight) between the stimulation-on and -off groups by using the log-rank test. The number of rotations and food intake were not analyzed as a function of time because it was not straightforward to describe this relationship with an appropriate parametric function. Indeed, circadian activity patterns varied considerably due to periods of sleep and food intake. Also, the group sizes were rather small to analyze the mean daily food intake and number of rotations in function of time. Therefore, we averaged the number of rotations and food intake for each animal and compared the mean number of rotations and food intake using Wilcoxon tests without taking time into account.

\section{Results}

\section{Positioning of Electrode Tips}

Based on the stereotactic rat atlas of Paxinos and Wat- son, ${ }^{34}$ all electrode tips were located in the lateral hypothalamus except in 1 rat in the acute experiment, in which the electrode tip was located in the supraoptic chiasm. A schematic representation of the position of the electrode tips is depicted in Figs. 1 and 2.

\section{Submaximal and Maximal Stimulation Amplitude}

At submaximal stimulation amplitudes, some rats displayed exploratory behavior, walking criss-cross around in the cage, sniffing at the floor and the walls, and sometimes rearing with both forepaws on the walls of the cage. In other rats, seemingly automated locomotor stepping with elevated forepaws, resembling marching, was observed. Finally, some rats commenced rapid locomotion in the periphery of the cage, often interrupted by upright postures and sometimes even jumping as if they wanted to escape out of the cage. Because food was absent during testing for maximal stimulation amplitude, eating was not observed. At maximal stimulation amplitudes, these behaviors were exaggerated and clearly interfered with normal behavior. 


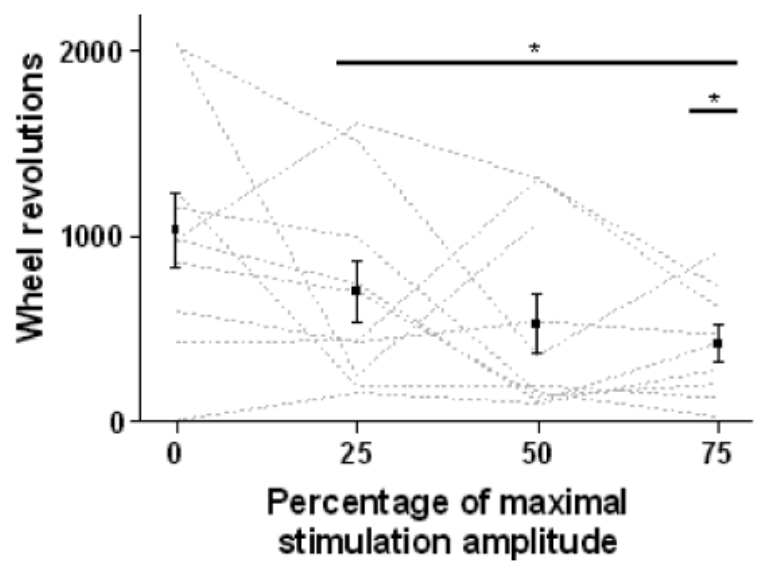

FIG. 3. Graph showing the number of wheel revolutions at 0,25 , 50 , and $75 \%$ of the maximal stimulation amplitude during the 2hour period preceding the feeding period in the acute experiment. Raw data for each animal are shown as dotted gray lines with mean \pm standard deviation superimposed in black. The asterisk indicates significant differences between the combined stimulated group $(25,50$, and $75 \%$ of the maximal stimulation amplitude combined) compared with $0 \%$ of the maximal stimulation amplitude $(\mathrm{p}<$ 0.04 ), and the group that was stimulated at $75 \%$ of the maximal stimulation amplitude compared with $0 \%(\mathrm{p}<0.04)$.

\section{Acute Experiment}

Raw data on the number of rotations and food intake for the different percentages of the maximal stimulation amplitude are represented respectively in Figs. 3 and 4. One value was missing unrelated to the intervention (electrical stimulation).

Overall electrical stimulation in the lateral hypothalamus $(25,50$, and $75 \%$ of the maximal stimulation amplitude combined) induced a significant decrease of the number of rotations compared with $0 \%$ of the maximal stimulation amplitude $(-467 \pm 192$, estimated difference (standard error, $\mathrm{p}<0.04)$, however, without affecting food intake $(-0.02 \pm 0.60 \mathrm{~g}, \mathrm{p}<0.97)$.

Figure 3 gives the impression that the decrease in the number of rotations is amplitude-dependent; however, no significant difference between the number of rotations at 25,50 and $75 \%$ of the maximal stimulation amplitude was observed ( $\mathrm{p}<0.39$ ). Likewise, no significant difference was observed between food intake at 25,50 , and $75 \%$ of the maximal stimulation amplitude ( $\mathrm{p}<0.74$; Fig. 4).

Paired Wilcoxon tests revealed a significant decrease of the number of rotations at $75 \%$ of the maximal stimulation amplitude compared with $0 \%(\mathrm{p}<0.04)$, but no difference for food intake $(\mathrm{p}<0.93)$. However, if the data obtained from the rat with 1 electrode tip in the supraoptic chiasm were omitted, overall electrical stimulation no longer had a significant effect on the number of rotations ( $\mathrm{p}<0.07$ ). Likewise, the number of rotations at $75 \%$ compared with $0 \%$ of the maximal stimulation amplitude was no longer significantly different $(\mathrm{p}<0.08)$.

\section{Chronic Experiment}

Two rats from the stimulation-on group were excluded because their body weight remained constant during 5 con-

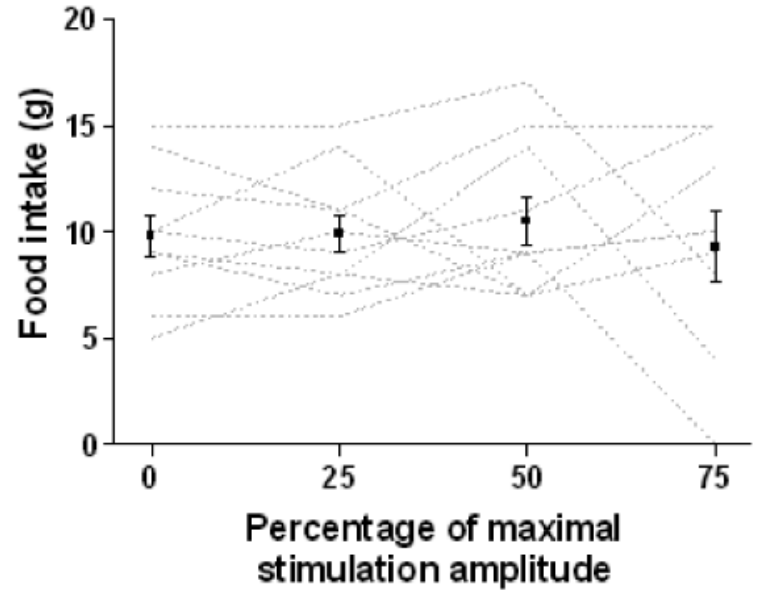

FIG. 4. Graph showing the food intake (in g) at $0,25,50$, and $75 \%$ of the maximal stimulation amplitude during the 1.5 -hour feeding period in the acute experiment. Raw data for each animal are shown as dotted gray lines with mean \pm standard deviation superimposed in black.

secutive days before reaching $85 \%$ of their initial body weight. For the remaining rats, the number of days after reaching $85 \%$ of the initial body weight until the end of the experiment (as defined above) was $4.5 \pm 3.9$ days in the stimulation-on group and $4.8 \pm 2.0$ days in the stimulationoff group. Of note, body weight of 1 rat in the stimulationon group remained constant during 5 consecutive days. Therefore this rat was considered a survivor (censored). The log-rank test revealed no significant differences for the number of days between both groups ( $\mathrm{p}<0.57$ ).

Electrical stimulation neither had an effect on the number of wheel revolutions preceding the feeding period (stimulation on $111 \pm 67$ rotations/hour, stimulation off $128 \pm 87$ rotations/hour, mean \pm standard deviation; $\mathrm{p}<$ 0.30, Fig. 5) nor on the food intake per day during the 1.5hour feeding period (stimulation on $5.4 \pm 4.5 \mathrm{~g}$, stimulation off $7.8 \pm 4.1 \mathrm{~g}$, mean \pm standard deviation; $\mathrm{p}<0.22$ [Fig. 6]).

\section{Discussion}

\section{Activity-Based Anorexia Model}

Rats in the activity-based anorexia model, which were fed at libitum for 1.5 hours a day and had continuous access to a running wheel, were not able to sustain their body weight and even died. ${ }^{14,39}$ Only a minority of the rats were resistant to this procedure and survived (8-12\% of our sample). In the current experiment, the daily distance in the running wheel was up to $10.7 \mathrm{~km}$ after 1 to 2 weeks in some rats, with a daily food intake on average between 5 and 15 $\mathrm{g}$ for the acute and chronic experiment, both in accordance with previous experiments. ${ }^{9}$

\section{Validity of the Model}

Animal models for psychiatric disorders provide a valuable tool for developing new treatments. The models need to be subjected to strict validating criteria, including face, predictive, and construct validity. ${ }^{49}$ Johansen and Schalling $^{21}$ concluded in their review that the activity-based 


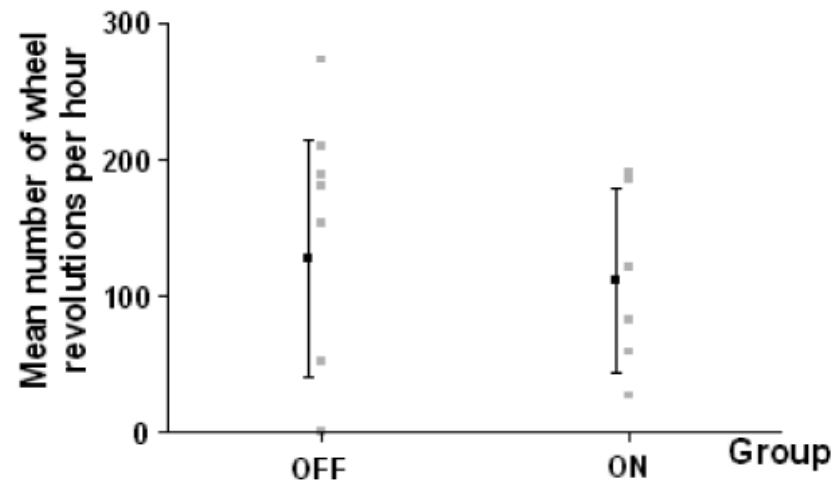

FIG. 5. Graph showing the number of wheel revolutions in the chronic experiment for the stimulation-on and -off groups. For each animal, the number of revolutions over days is averaged and presented as gray boxes with superimposed means \pm SD in black.

anorexia model has a better face and construct validity than other models for anorexia nervosa. The authors were, however, reserved in attributing predictive validity to the models due to the lack of potent pharmacological agents for treating patients with anorexia nervosa. The effect of cyproheptadine, which has some modest benefit during the weight restoration phase in patients with anorexia nervosa ${ }^{36}$ has not been evaluated in the activity-based anorexia model. Fluoxetine has a comparable effect in patients with anorexia nervosa and in the activity-based anorexia model: during the weight maintenance phase the relapse rate may decrease in patients with anorexia nervosa ${ }^{36}$ and it induces a decrease in wheel running and an increase in food intake in the activity-based anorexia model. ${ }^{1,2}$ Also, increasing the environmental temperature in patients with anorexia nervosa as well as in rats in the activity-based anorexia model was reported to have beneficial effects. ${ }^{13,43}$ Finally, discriminant validity was raised as a fourth additional criterion for animal models for psychiatric disorders. ${ }^{12}$ The model should specifically point to anorexia nervosa as distinct from different psychiatric disorders. The activity-based anorexia model was mainly used as a model for anorexia nervosa, although it has also been used as a model for gastric ulcers (as reported by Paré and Temple ${ }^{33}$ ) and has been proposed as a model for obsessive-compulsive disorder. ${ }^{1}$ However, this does not depreciate the value of the activity-based anorexia model as a model for anorexia nervosa because ulcers are present in $16 \%$ of the patients with anorexia nervosa, ${ }^{13}$ and obsessive-compulsive disorder is related to and often comorbid with anorexia nervosa. ${ }^{8,11,20,22,35,44}$

\section{Neuroanatomy of the Lateral Hypothalamus}

The lateral hypothalamus plays a key role within a distributed neural network that controls the regulation of food intake and body weight. ${ }^{6}$ It has efferent projections to the entire cortical mantle, hippocampus, extended amygdala, basal ganglia and thalamus, the midbrain and pons, the brainstem and spinal cord, as well as most other nuclei of the hypothalamus. Afferents originate from various corti$\mathrm{cal} /$ limbic areas such as the prefrontal/orbitofrontal, insular, and olfactory cortex, amygdalar cortex, hippocampus, and

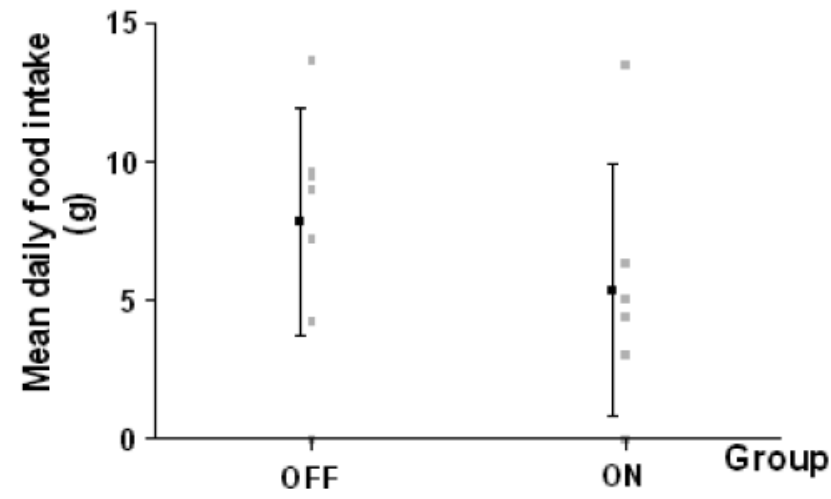

FIG. 6. Graph showing the food intake (in g) in the chronic experiment for the stimulation-on and -off group. For each animal, the number of revolutions over days is averaged and presented as gray boxes with superimposed means \pm SDs in black.

the ventral striatum, including the nucleus accumbens. Most of the connections to the brainstem and midbrain areas are reciprocal.

\section{The Hypothalamus and the Activity-Based Anorexia Model}

Alterations in local neurotransmitter levels provide direct evidence for the involvement of the hypothalamus in the activity-based anorexia model. Noradrenergic, ${ }^{38,48}$ histaminergic, ${ }^{10}$ and dopaminergic ${ }^{17}$ neuronal activity in the hypothalamus are significantly enhanced while serotonergic metabolism was decreased. ${ }^{17}$ Additional indirect evidence for the involvement of the hypothalamus comes from the increased activity of the hypothalamic-pituitary adrenal axis in this model. ${ }^{4,7}$

Some authors evaluated the effect of specific interventions in subregions of the hypothalamus on behavior in the activity-based anorexia model. A kainic lesion in the ventromedial hypothalamus decreased activity in the running wheel and body weight loss was decelerated, but this did not affect food intake. ${ }^{19}$ On the contrary, a lesion in the suprachiasmatic nucleus of the hypothalamus had no effect and local administration of clonidine, an $\alpha 2$ noradrenaline receptor agonist, in the paraventricular hypothalamus even decreased food intake and survival in rats in the activitybased anorexia model. In normal rats, however, a recent study demonstrated weight loss after chronic electrical stimulation in the lateral hypothalamus. ${ }^{41}$

\section{Acute Experiment}

Activity. At submaximal and maximal electrical stimulation in the lateral hypothalamus, we observed an initiation of automated stepping and increased exploratory and escape behavior, which is in agreement with the observations of other authors. ${ }^{23,42}$ The descending course of the pathway mediating locomotion elicited by electrical stimulation of the lateral hypothalamus is known to involve the ventral tegmental area and the red nucleus in the anteromedial ventral tegmentum and the pedunculopontine nucleus, the pontis oralis nucleus, and the region around the superior cerebellar peduncle more posterior in the mesencephalon. ${ }^{24}$

Electrical stimulation in the lateral hypothalamus in the activity-based anorexia experiment significantly reduced 
wheel-running. However, our findings were no longer significant after omission of the data of 1 rat. Of note, the electrode tip was on one side in the supraoptic chiasm but abutting the lateral hypothalamus $(<0.2 \mathrm{~mm}$, Fig. 1$)$, and the current likely spread into the lateral hypothalamus. Moreover, the electrode tip on the other side was well positioned in the lateral hypothalamus. Likely, a decrease in power may explain our findings.

Food Intake. Rather unexpectedly, we observed no significant effect of acute electrical stimulation in the lateral hypothalamus on food intake in rats in the activity-based anorexia model. Nevertheless, electrical stimulation in the lateral hypothalamus may induce feelings of hunger, as reported by obese patients during acute electrical stimulation experiments in the lateral hypothalamus before making a lesion. ${ }^{37}$ In rats, electrical stimulation in the lateral hypothalamus induces an eating response and a significant increase in the amount of food intake. ${ }^{26,27}$ The target for electrical stimulation in our experiments was derived from one of those experiments in which a large number of rats responded with an increase in food intake. It is also unlikely that the severe state of emaciation in the activity-based anorexia model is the reason that electrical stimulation had no effect on our experiment. Indeed, it has been demonstrated that electrical stimulation in the lateral hypothalamus in rats that were deprived of food for 72 hours has a larger effect on food intake than electrical stimulation or deprivation separately. ${ }^{46}$ Likewise, in rats with amphetamine-induced appetite suppression, electrical stimulation in the lateral hypothalamus increased food intake. ${ }^{47}$

\section{Chronic Experiment}

Both the activity in the running wheel and food intake were unaffected in our chronic experiment, although we observed a decrease in activity in our acute experiment. It may be that electrical stimulation has only a temporary effect on activity or that the stimulation amplitude needs to be raised to sustain the effect on activity. However, the latter argument is contradicted by the findings of our acute experiment, in which we observed no difference in activity between electrical stimulation with 25,50 , or $75 \%$ of the maximal stimulation amplitude. Another explanation is that the activity in the running wheel in our acute experiment was replaced by activity in the rest of the cage with no effect on global activity as the net result. Given that we did not record activity in the rest of the cage, this hypothesis cannot be excluded.

As both hyperactivity and a decrease in food intake are the main determinants for a bad outcome in the activitybased anorexia model, we observed no effect on survival time after chronic electrical stimulation in the lateral hypothalamus.

\section{Neurosurgery in Patients With Anorexia Nervosa}

In several patients suffering from severe anorexia nervosa, the effect of surgical lesions in different brain regions (for example, leukotomy, ${ }^{28}$ dorsomedial thalamotomy, ${ }_{15}^{15}$ and so on) was investigated, and most of the studies demonstrated a poor outcome on core symptoms. Moreover, lesions are irreversible, a disadvantage in case severe side effects occur. Therefore, we opted for investigating the ef- fects of electrical brain stimulation, which is a reversible technique. As far as we know, electrical brain stimulation in only 1 patient suffering from anorexia nervosa has been published. ${ }^{16}$ She had a 12-year history of anorexia nervosa, was completely amenorrheic, and had to be tube-fed. Electrical stimulation on the cerebellar cortex induced a pleasant and relaxed feeling, and reduced associated compulsive rituals. Her menstrual cycle returned to normal but she still continued to refuse eating.

\section{Study Limitations}

In the current experiments, we preferred to work with female rats because the incidence of anorexia nervosa in humans is more than 10 times higher in females than males. However, in female rats, food intake and wheel running fluctuated with the estrous cycle, resulting in a higher dayto-day variability ${ }^{3}$ and decreasing the likeliness to observe small effect sizes. We did not synchronize the estrous cycle with the day of introduction in the activity-based anorexia model.

The size of the groups is rather limited, especially the size of the stimulation-on group in the chronic experiment (6 rats). However, the findings of the acute and the chronic experiments are in agreement with each other and are not indicative for major differences.

We did not record the activity of the rats in the rest of the cages (for example, by measuring the number of infrared beam crossings); it is unknown whether the decrease in activity observed in our acute experiment is generalized.

\section{Conclusions}

Electrical stimulation in the lateral hypothalamus decreased hyperactivity during acute but not chronic electrical stimulation, with no effect on food intake and survival time. Although extrapolation of findings in animal experiments to humans is not straightforward, especially in psychiatric disorders, we do not expect that electrical stimulation in the lateral hypothalamus will affect symptoms in patients suffering from anorexia nervosa.

\section{Disclaimer}

The authors do not report any conflict of interest concerning the materials or methods used in this study or the findings specified in this paper.

\section{Acknowledgment}

We thank Dr. S. Fieuws of the Biostatistical Centre of the Katholieke Universiteit Leuven for his support in the statistical analyses.

\section{References}

1. Altemus M, Glowa JR, Galliven E, Leong YM, Murphy DL: Effects of serotonergic agents on food-restriction-induced hyperactivity. Pharmacol Biochem Behav 53:123-131, 1996

2. Altemus M, Glowa JR, Murphy DL: Attenuation of food-restriction-induced running by chronic fluoxetine treatment. Psychopharmacol Bull 29:397-400, 1993

3. Anantharaman-Barr HG, Decombaz J: The effect of wheel running and the estrous cycle on energy expenditure in female rats. Physiol Behav 46:259-263, 1989

4. Aravich PF, Rieg TS, Lauterio TJ, Doerries LE: Beta-endorphin and dynorphin abnormalities in rats subjected to exercise and 
restricted feeding: relationship to anorexia nervosa? Brain Res 622:1-8, 1993

5. Ben-Tovim DI, Walker K, Gilchrist P, Freeman R, Kalucy R, Esterman A: Outcome in patients with eating disorders: a 5-year study. Lancet 357:1254-1257, 2001

6. Berthoud HR: Multiple neural systems controlling food intake and body weight. Neurosci Biobehav Rev 26:393-428, 2002

7. Burden VR, White BD, Dean RG, Martin RJ: Activity of the hypothalamic-pituitary-adrenal axis is elevated in rats with activity-based anorexia. J Nutr 123:1217-1225, 1993

8. Collier DA, Arranz MJ, Li T, Mupita D, Brown N, Treasure J: Association between 5-hT2A gene promoter polymorphism and anorexia nervosa. Lancet 350:412, 1997

9. Dixon DP, Ackert AM, Eckel LA: Development of, and recovery from, activity-based anorexia in female rats. Physiol Behav 80: 273-279, 2003

10. Endou M, Yanai K, Sakurai E, Fukudo S, Hongo M, Watanabe T: Food-deprived activity stress decreased the activity of the histaminergic neuron system in rats. Brain Res 891:32-41, 2001

11. Enoch MA, Kaye WH, Rotondo A, Greenberg BD, Murphy DL, Goldman D: 5-hT2A promoter polymorphism-1438G/A, anorexia nervosa, and obsessive-compulsive disorder. Lancet 351:1785-1786, 1998

12. Geyer M, Markou A: Animal models of psychiatric disorders, in Bloom FE, Kupfer DJ (eds): Psychopharmacology: Fourth Generation of Progress. New York: Raven, 1995, pp 787-798

13. Gutiérrez E, Vázquez R, Boakes RA: Activity-based anorexia: ambient temperature has been a neglected factor. Psychon Bull Rev 9:239-249, 2002

14. Hall JF, Hanford PV: Activity as a function of a restricted feeding schedule. J Comp Physiol Psychol 47:362-363, 1954

15. Hassler R, Dieckmann G: Stereotaxic treatment of compulsive and obsessive symptoms. Confin Neurol 29:153-158, 1967

16. Heath RG, Llewellyn RC, Rouchell AM: Brain mechanisms in psychiatric illness: rationale for and results of treatment with cerebellar stimulation, in Hitchcock ER, Ballantine J, Meyerson BA (eds): Modern Concepts in Psychiatric Surgery: Elsevier, North-Holland Biomedical Press, 1979, pp 77-84

17. Hellhammer DH, Rea MA, Bell M, Belkien L: Activity-wheel stress: effects on brain monoamines and the pituitary-gonadal axis. Neuropsychobiology 11:251-254, 1984

18. Hoek HW, van Hoeken D: Review of the prevalence and incidence of eating disorders. Int J Eat Disord 34:383-396, 2003

19. Iwamoto Y, Nishihara M, Takahashi M: VMH lesions reduce excessive running under the activity-stress paradigm in the rat. Physiol Behav 66:803-808, 1999

20. Jarry JL, Vaccarino FJ: Eating disorder and obsessive-compulsive disorder: neurochemical and phenomenological commonalities. J Psychiatry Neurosci 21:36-48, 1996

21. Johansen J, Schalling M: Animals models of eating disorders, in D'Haenen HAH, den Boer JA, Willner P (eds): Biological Psychiatry. New York: Wiley, 2002, pp 1117-1126

22. Kaye WH, Weltzin TE, Hsu LKG, Bulik C, McConaha C, Sobkiewicz T: Patients with anorexia nervosa have evaluated scores on the Yale-Brown obsessive compulsive scale. Int J Eating Disord 12:57-62, 1992

23. Lammers JH, Kruk MR, Meelis W, van der Poel AM: Hypothalamic substrates for brain stimulation-induced patterns of locomotion and escape jumps in the rat. Brain Res 449:294-310, 1988

24. Levy DI, Sinnamon HM: Midbrain areas required for locomotion initiated by electrical stimulation of the lateral hypothalamus in the anesthetized rat. Neuroscience 39:665-674, 1990

25. Mayberg HS, Lozano AM, Voon V, McNeely HE, Seminowicz D, Hamani C, et al: Deep brain stimulation for treatment-resistant depression. Neuron 45:651-660, 2005

26. Mogenson GJ, Gentil CG, Stevenson JA: Feeding and drinking elicited by low and high frequencies of hypothalamic stimulation. Brain Res 33:127-137, 1971

27. Mogenson GJ, Wu M: Neuropharmacological and electrophysiological evidence implicating the mesolimbic dopamine system in feeding responses elicited by electrical stimulation of the medial forebrain bundle. Brain Res 253:243-251, 1982

28. Morgan JF, Crisp AH: Use of leucotomy for intractable anorexia nervosa: a long-term follow-up study. Int J Eat Disord 27: 249-258, 2000

29. Nuttin B, Cosyns P, Demeulemeester H, Gybels J, Meyerson B: Electrical stimulation in anterior limbs of internal capsules in patients with obsessive-compulsive disorder. Lancet 354:1526, 1999

30. Nuttin B, Gybels J, Cosyns P, Gabriels L, Meyerson B, Andreewitch S, et al: Deep brain stimulation for psychiatric disorders. Neurosurg Clin N Am 14:xv-xvi, 2003

31. Nuttin BJ, Gabriëls LA, Cosyns PR, Meyerson BA, Andreewitch S, Sunaert SG, et al: Long-term electrical capsular stimulation in patients with obsessive-compulsive disorder. Neurosurgery 52: 1263-1274, 2003

32. O'Brien KM, Vincent NK: Psychiatric comorbidity in anorexia and bulimia nervosa: nature, prevalence, and causal relationships. Clin Psychol Rev 23:57-74, 2003

33. Paré WP, Temple LJ: Food deprivation, shock stress and stomach lesions in the rat. Physiol Behav 11:371-375, 1973

34. Paxinos G, Watson C: The Rat Brain in Stereotactic Coordinates, ed 2. Sydney: Academic Press, 1986

35. Pigott TA, Altemus M, Rubenstein CS, Hill JL, Bihari K, L'Heureux F, et al: Symptoms of eating disorders in patients with obsessive-compulsive disorder. Am J Psychiatry 148:1552-1557, 1991

36. Powers PS, Santana C: Available pharmacological treatments for anorexia nervosa. Expert Opin Pharmacother 5:2287-2292, 2004

37. Quaade F, Vaernet K, Larsson S: Stereotaxic stimulation and electrocoagulation of the lateral hypothalamus in obese humans. Acta Neurochir (Wien) 30:111-117, 1974

38. Rea MA, Hellhammer DH: Activity wheel stress: changes in brain norepinephrine turnover and the occurrence of gastric lesions. Psychother Psychosom 42:218-223, 1984

39. Routtenberg A: "Self-starvation" of rats living in activity wheels: adaptation effects. J Comp Physiol Psychol 66:234238, 1968

40. Routtenberg A, Kuznesof AW: Self-starvation of rats living in activity wheels on a restricted feeding schedule. J Comp Physiol Psychol 64:414-421, 1967

41. Sani S, Jobe K, Smith A, Kordower JH, Bakay RA: Deep brain stimulation for treatment of obesity in rats. J Neurosurg 107: 809-813, 2007

42. Sinnamon HM, Karvosky ME, Ilch CP: Locomotion and head scanning initiated by hypothalamic stimulation are inversely related. Behav Brain Res 99:219-229, 1999

43. Södersten P, Bergh C, Ammar A: Anorexia nervosa: towards a neurobiologically based therapy. Eur J Pharmacol 480:67-74, 2003

44. Sorbi S, Nacmias B, Tedde A, Ricca V, Mezzani B, Rotella CM: 5-hT2A promoter polymorphism in anorexia nervosa. Lancet 351:1785, 1998

45. Støving RK, Hangaard J, Hansen-Nord M, Hagen C: A review of endocrine changes in anorexia nervosa. J Psychiatr Res 33: 139-152, 1999

46. Tenen SS, Miller NE: Strength of electrical stimulation of lateral hypothalamus, food deprivation, and tolerance for quinine in food. J Comp Physiol Psychol 58:55-62, 1964

47. Thode WF, Carlisle HJ: Effect of lateral hypothalamic stimulation on amphetamine-induced anorexia. J Comp Physiol Psychol 66:547-548, 1968 


\section{Lateral hypothalamus stimulation in the activity-based anorexia model}

48. Tsuda A, Tanaka M, Kohno Y, Nishikawa T, Iimori K, Nakagawa $\mathrm{R}$, et al: Marked enhancement of noradrenaline turnover in extensive brain regions after activity-stress in rats. Physiol Behav 29: 337-341, 1982

49. Willner P: Validation criteria for animal models of human mental disorders: learned helplessness as a paradigm case. Prog Neuropsychopharmacol Biol Psychiatry 10:677-690, 1986

50. Zipfel S, Löwe B, Reas DL, Deter HC, Herzog W: Long-term prognosis in anorexia nervosa: lessons from a 21-year follow-up study. Lancet 355:721-722, 2000

Manuscript submitted April 10, 2008.

Accepted April 21, 2008.

The data in the current study were presented as part of the Ph.D. thesis manuscript of Dr. Kris van Kuyck (Electrical brain stimulation in psychiatric disorders, Kris van Kuyck, ISBN: 97890 58675545, 2006, Leuven: Leuven University Press, pp 83-95) Sources of support: Research Fund Katholieke Universiteit Leuven (project VIS/02/007 and OT/03/57), the Fonds voor Wetenschappelijk Onderzoek-Vlaanderen (G.0598.06), and the Institute for the Promotion of Innovation by Science and Technology in Flanders (SBO50151). M. Welkenhuysen is also a doctoral fellow of the Institute for the Promotion of Innovation by Science and Technology in Flanders (No. 61236).

Address correspondence to: Bart Nuttin, M.D., Ph.D., Department of Experimental Neurosurgery and Neuroanatomy, U.Z. Herestraat 49 bus 7003, B-3000 Leuven, Belgium. email: bart. nuttin@uz.kuleuven.be. 